\title{
Autoestima y ansiedad en los adolescentes
}

\author{
M. ${ }^{a}$ Ángeles Hernández Prados. Universidad de Murcia \\ Lorena Belmonte García.Universidad de Murcia \\ M. ${ }^{a}$ de las Mercedes Martínez Andreo. Universidad de Murcia
}

\author{
Recepción: 20.07.2018 | Aceptado: 15.09.2018 \\ Correspondencia a través de ORCID: M. ${ }^{a}$ Ángeles Hernández iD 0000-0002-3617-2015X \\ Citar: Hernández Prados, MA., Belmonte García, L. y Martínez Andreo, MM. (2018). Autoestima \\ y ansiedad en los adolescentes. ReiDoCrea, 7, 269-278.
}

\begin{abstract}
Resumen: La adolescencia entendida como la etapa de transición a la vida adulta, se caracteriza por el cambio, especialmente emocional que pueden derivar en problemas de autoestima y respuestas de ansiedad desadaptativas ante determinadas situaciones. Los estudios previos desvelan efectos en el rendimiento educativo y en la convivencia, siendo esencial el papel de la escuela y la familia para la adquisición de estrategias preventivas. Metodo: Con la finalidad de valorar cual es el nivel de autoestima de los estudiantes a través del RSE (autoestima de Rosenberg) y analizar cómo éste influye en el nivel de ansiedad que se determinó mediante el STAI (State Trait Anxiety Inventory), se llevó a cabo en el curso académico 2015-2016 una investigación de tipo descriptivo-correlacional, en la que participaron 97 alumnos de Educación Secundaria Obligatoria. Resultados: El análisis estadístico refiere que la mayor parte de la muestra $(98,9 \%)$, manifiesta niveles medios y altos de autoestima. Con respecto a la ansiedad estado y rasgo, los valores se sitúan en gran medida en un nivel medio, $(55,7 \%)$ y $(60,8 \%)$, respectivamente. Conclusiones: La relación entre autoestima y ansiedad, es negativa y estadísticamente significativa, observando que a una mayor autoestima los estudiantes presentan menor ansiedad estado y rasgo.
\end{abstract}

Palabras clave: Ansiedad | Autoestim

\section{Self-esteem and anxiety of the teenagers}

Abstract: Background:The adolescence as the stage of transition to the adulthood is characterized by the change. Those, go through a big range of emotional states, which can bring with them the development of self-esteem problems and answers poorly adapted to particular situations. These concepts, especially that of self-esteem, are characterized by their ambiguity. There isn't consensus in the definition. But, this doesn't exempt the educational contexts from working on them, because there are many benefits for students: happiness and mental health, greater leadership, relaxation and self-control, school performance, better social relationships and communicate more fluently. Previous studies reveal effects on educational performance and coexistence. The educational context is alongside the family context, a reference in the training and acquisition of strategies for those problematic situations which at the same time can affect the academic performance. Method:With the aim of valuing the level of self-esteem of the students through of RSE (Rosenberg's self-esteem) and analyzing the influence of this in the level of anxiety that it was determined through STAI (State Trait Anxiety Inventory), conducted a descriptivecorrelational research in the academic year 2015-2016, in which 97 students of Compulsory Secondary Education participated. Results: The statistical analysis refers that the major part of the sample $(98,9 \%)$, manifest high and medium levels of self-esteem. However, there are no differences according to gender and age. Regarding the state and feature anxiety, the values tend to be in a medium level, $(55,7 \%)$ and $(60,8 \%)$, respectively. Conclusions:The relation/link between self-esteem and anxiety is negative and statistically meaningful, observing that the higher the self-esteem, the lower state and feature anxiety shown by the students.

Keywords: Anxiety | Self-esteem

\section{Introducción}

Concebir la adolescencia como etapa de grandes cambios psico-fisicos y sociales, así como una crisis de identidad constante, resulta habitual tanto en la producción científica como en el lenguaje cotidiano. La vulnerabilidad a la que se expone la autoestima derivada del fuerte protagonismo que los adolescentes conceden a las 
relaciones entre iguales contribuyó potencialmente al incremento de los estudios sobre la misma, iniciándose por primera vez con Rosenberg en 1965 y poco después por Erikson (1968), quién puso de manifiesto que la pérdida de identidad del adolescente genera hostilidad en el contexto familiar, lo que ha llevado a definir la adolescencia como una etapa de rebeldía.

El concepto de autoestima ha estado tradicionalmente unido al de autoconcepto, de hecho, las primeras definiciones son poco claras y difusas en lo que respecta a la distinción de ambos términos, empleando ambos constructos indistintamente para hacer referencia al conocimiento que el ser humano tiene de sí mismo. Confirmando la relación entre ambos, Musitu (1996) define la autoestima como el concepto que uno tiene de sí mismo, según una serie de cualidades que uno se atribuye. Sin embargo, autores como Garaigordobil y Durá (2006) definen el autoconcepto como los aspectos cognitivos del conocimiento de uno mismo y la autoestima como los aspectos valorativos o afectivos. En este sentido, la autoestima se encuentra vinculada al autoconcepto tanto al ideal propio como al deseado por los otros significativos, de modo que incluye lo que me gustaría ser (por tener un gran valor e importancia para mí) y lo que a los demás les gustaría que yo fuese (por el valor que ello tiene para aquéllos), e incluye contenidos tanto de naturaleza social como privada (GonzálezPienda, Nuñez Pérez, Glez Pumariega y García García, 1999).

Por otra parte, la definición de Branden (1993) considera que el ser humano no es capaz de expresar todo su potencial sin una sana autoestima y sin una valoración y confianza en sí mismos, y define este concepto como la experiencia de ser aptos para la vida y para las necesidades de la vida, incluyendo la importancia de confiar en nuestra capacidad de pensar y de afrontar los desafíos de la vida. Posteriormente en su libro "Los seis pilares de la autoestima" (1995), Branden introduce el concepto de falsa autoestima como la apariencia irreal de ser eficaz, respetado, seguro y estimado, que se utiliza como protección para disminuir la ansiedad y proporcionarnos un sentido falso de seguridad para aliviar las necesidades de una autoestima auténtica.

No hay, por ahora, una posición unánime respecto a qué se considera autoestima. Como afirma Smelser (1989, p. 9): "disponemos de una percepción bastante firme de lo que significa el término autoestima, tal y como se revela mediante nuestra propia introspección y la observación de la conducta ajena. Pero cuesta mucho expresar tal comprensión en términos precisos", por la multiplicidad de términos a los que se asocia: conciencia de si mismo, autoimagen, autopercepción, representación de sí, autoconcepto (Montoya y Landero, 2013). Por ello, aunque generalmente se sostiene que para abordar racionalmente los problemas en educación se exige definir los términos en cuestión y clarificar la naturaleza del problema, se reconoce la necesidad de trabajar la autoestima en lo diferentes contextos educativos a pesar de su imprecisión lingüística, por la multitud de beneficios que ésta conlleva en el desarrollo del ser humano. De hecho, para González y Turón (1992) la autoestima es la clave del éxito o el fracaso para comprendernos y comprender a los demás, ya que ésta afecta de manera considerable en todos los aspectos de nuestra experiencia, además de estar formada por dos componentes: un sentimiento de capacidad personal y un sentimiento de valía personal. De ello se desprende la necesidad de alcanzar una autoestima óptima, siendo consciente de las propias posibilidades y limitaciones, así como dar los pasos para conseguir en la medida nuestras verdaderas metas, además de mantener un mejor funcionamiento global de la persona (Kernis, 2003).

La autoestima es esencial en el desarrollo personal, social y escolar, ya que proporciona confianza y seguridad en las personas. De modo que, la formación personal, la construcción de la felicidad y salud mental, el establecimiento de 
relaciones adecuadas con el entorno, mayor relajación y autocontrol, y el desarrollo del aprendizaje y por ende del rendimiento escolar, son algunos de los aspectos que depende de una buena autoestima (Fernández, y Extremera, 2002; Ciarrochi, Chan y Bajgar, 2001; Rodríguez y Caño, 2012). Desde la perspectiva escolar, los alumnos que presentan altos niveles de autoestima muestran un mayor liderazgo, mejores relaciones sociales y se comunican más fluidamente (Aldana, 2012). En contraposición, niveles bajos de autoestima han sido asociados con "sentimientos de aislamiento, apatía y pasividad mientras que la alta autoestima se relaciona con personas más activas, menos ansiosas y con mejores relaciones sociales", afirman Cándido et al (2016) tras la consulta de varios estudios previos.

La autoestima es un elemento primordial nutrido de las experiencias que vamos incluyendo en nuestro bagaje personal, así como de opiniones y experiencias que los demás vierten sobre nosotros, en cualquier etapa de nuestra vida, formando así un pilar fundamental para nuestro desarrollo emocional y personal. Esas experiencias y opiniones que los demás muestran sobre nosotros, pueden ser todavía más importantes en la adolescencia, ya que, en esta etapa aumenta la preocupación por los cambios que conlleva pasar de la niñez a la vida adulta, además de mostrar confusión en relación a su propia imagen y lo que los demás opinan y esperan de ellos.

Por otro lado, la ansiedad entendida como un rasgo de la personalidad, por lo que cada persona la vivencia de una manera u otra en situaciones similares o diferentes (Endler, 1975), como la respuesta del organismo ante un estímulo determinado, la cual desencadena una reacción que activa el sistema nervioso (Bobes, et al.2001) ha sido considerada, desde el ámbito escolar, como una importante área de investigación, sobre todo en lo referente a los resultados académicos, aunque ha estado algo desatendida. Cada vez más escolares presentan problemas emocionales, los desórdenes de ansiedad afectan aproximadamente entre el 13 y el $17 \%$ de los jóvenes (Kashani y Orvaschel 1990). En la ansiedad, se da una combinación tanto de síntomas cognitivos como fisiológicos, los cuales se manifiestan en una continua reacción de sobresalto, donde el individuo trata de buscar una solución al peligro, pero no siempre es buscado de la mejor manera (Sierra, Ortega y Zubeida, 2003).

La mayoría de los estados ansiosos de esta etapa de la adolescencia se relacionan con el bajo rendimiento académico y el fracaso escolar, pero existen otras esferas personales, relacionales, emocionales y convivenciales a las que también afectan. De modo que, si la ansiedad es intensa, el estudiante muestra excesiva sensibilidad y temores, lo que dará lugar a una disminución de la autoestima y del autoconcepto (Jadue, 2001). La ansiedad depende en gran medida de la idea que el alumno tiene sobre la tarea que le es demandada y exigida, y como ésta está relacionada con su capacidad de comprensión y de control que en esos momentos tiene sobre sí mismo. Cuando el alumno piensa que no está capacitado para aquello que se le demanda y que es más de lo que él puede rendir, aparece en él una situación de humillación, de rechazo hacia el ámbito académico. Por todo ello, este estudio se propone como finalidad analizar la relación que existe entre la ansiedad y la autoestima en el alumnado de educación secundaria. 


\section{Aspectos metodológicos}

\section{Objetivos}

El objetivo general que nos planeamos con el presente trabajo es estudiar la relación entre ansiedad y autoestima en alumnos de Educación Secundaria Obligatoria. Para ello se plantean los siguientes objetivos específicos:

- Identificar los niveles de ansiedad (estado y rasgo), así como el nivel de autoestima en el alumnado de secundaria, en función del género y la edad

- Analizar si existe relación entre la ansiedad y autoestima en el nivel educativo estudiado.

\section{Participantes}

El número de participantes en este estudio esta compuesto por 97 estudiantes de ESO cuyas edades oscilan de los 12 a los 16 años, situándose la mayoría en los 14 años. Se trata de un estudio de caso único, por lo tanto la muestra ha sido extraída de un IES del municipio de Totana (Murcia) seleccionado atendiendo a los siguientes criterios: vinculación con el centro, proximidad geográfica, disponibilidad e interés del centro para participar en la investigación, así como con la implicación de los tutores en la recogida de la información. La distribución de la muestra por género $(50,5$ femenino y 49,5 masculino) y por curso $(24,74 \%$ de primero, $28,86 \%$ de segundo, $20,62 \%$ de tercero y $25,77 \%$ de cuarto) es proporcional y equitativa.

\section{Instrumentos}

Se trata de un estudio de carácter descriptivo transversal con datos de tipo cuantitativo. Se han utilizado dos instrumentos objetivos, tipo test con cuestiones cuya respuesta está basada en una escala tipo Likert, validados previamente en diversas investigaciones.

La primera prueba, el cuestionario State Trait Anxiety Inventory (STAI) desarrollado por Spielbelger, Gorsuch y Lushene (1970), evalúa el nivel de ansiedad estado-rasgo con un rango de intensidad de 0 a 3 ( $0=$ casi nunca/nada; $1=$ algo/a veces; $2=$ bastante/ a menudo y $3=$ mucho/casi siempre). Consta de 40 ítems divididos en dos subescalas de veinte items cada una de ellas para medir el nivel de ansiedad estado (estado emocional transitorio, caracterizado principalmente por una serie de sentimientos subjetivos, los cuales pueden ser percibidos) y el nivel de ansiedad rasgo (estado relativamente estable que caracteriza a las personas como propensas a percibir demasiadas situaciones como amenazadoras).

La segunda prueba, la escala de autoestima de Rosenberg, creada por dicho autor en 1965, está constituido por 10 ítems en una escala tipo Likert, basados en los sentimientos de respeto y aceptación de sí mismo/a, valorados del 1 al 4 según su grado de adecuación (1= muy de acuerdo, 2=de acuerdo, $3=$ en desacuerdo y 4= totalmente de acuerdo). El estudio psicométrico del la escala muestra una alta consistencia interna y satisfactoria fiabilidad temporal, además de apoyar la validez del instrumento y resaltar la facilidad de aplicación y sus aceptables características psicométricas (Vázquez, Jiménez, Vázquez, 2004). 


\section{Procedimiento}

Este estudio se desarrolla durante el segundo semestre del curso académico 20152016, en el marco de la asignatura Tesis Final de Máster en la Facultad de Educación de la Universidad de Murcia, en el Máster de Formación del Profesorado en Educación Secundaria Obligatoria y Bachillerato. Tras la consulta de las fuentes, se delimitaron los objetivos e instrumentos del estudio, se pidió permiso al equipo directivo y al departamento de orientación del centro educativo y se procedió a reparto y cumplimentación de los instrumentos anteriormente descritos.

\section{Análisis de datos}

Los datos se analizaron con el paquete estadístico IBM SPSS Statistics versión 19. Se ha realizado principalmente un análisis estadístico descriptivo de frecuencias, porcentaje, medias y desviación típica de las diferentes variables contempladas en la investigación (edad, sexo, nivel de autoestima, nivel de ansiedad estado y nivel de ansiedad rasgo). Así mismo, para saber el grado de prevalencia de autoestima y ansiedad de los participantes, se han establecido tres niveles de intensidad en la escala de autoestima (nivel bajo (0-13), nivel medio (14-27) y nivel alto (28-40)) y en el cuestionario STAI (nivel bajo (0-20), nivel medio (21-40) y nivel alto (41-60)), atendiendo a los criterios expuestos por los propios autores.

\section{Resultados}

En lo que respecta a la distribución de los participantes en función al nivel de ansiedad registrado, los datos recogidos en la tabla 1 muestran que la mayoría se sitúan en la zona intermedia tanto en la ansiedad estado como en la ansiedad rasgo, sin embargo, el número de alumnos que presentan una alta ansiedad rasgo es más elevado que los que presentan una alta ansiedad estado, lo que implica que la ansiedad estable es mayor que la transitoria, siendo el $22,9 \%$ de los alumnos muy propensos a percibir frecuentemente las situaciones como amenazadoras. Por otra parte en lo que refiere a los niveles de autoestima, las tres cuartas partes del alumnado presenta una alta autoestima.

Tabla 1

Porcentajes de los niveles de ansiedad y autoestima en el alumnado de ESO

\begin{tabular}{llll}
\hline Niveles & Ansiedad estado & Ansiedad rasgo & Autoestima \\
\hline Bajo & $37,1 \%$ & $16,49 \%$ & $1,0 \%$ \\
Medio & $55,7 \%$ & $60,82 \%$ & $21,6 \%$ \\
\hline Alto & $7,2 \%$ & $22,88 \%$ & $77,3 \%$ \\
\hline
\end{tabular}

Dado que la muestra no sigue una distribución normal, se ha aplicado estadística no paramétrica, concretamente la prueba $U$ de Mann-Whitney, lo que ha permitido comprobar que no existe relación estadísticamente significativa entre la ansiedad y el genero y la edad. De igual manera, sucede con la autoestima.

No obstante a nivel de centro, resultaba interesante comprobar la distribución del alumnado en ansiedad y autoestima en función de las variables sociodemográficas. Para ello se calcularon las siguientes tablas de contingencia, que permiten comprobar, aunque sea de forma no significativa, que el número de mujeres que se encuentran en 
niveles de ansiedad altos supera al de los hombres (tabla 2), mientras que en los niveles altos de autoestima predominan los hombres.

Tabla 2. Nivel de ansiedad-estado en función del género

\begin{tabular}{|c|c|c|c|c|c|c|c|c|c|}
\hline & \multicolumn{3}{|c|}{ NIVEL DE ANSIEDAD-ESTADO } & \multicolumn{3}{|c|}{ NIVEL DE ANSIEDAD-RASGO } & \multicolumn{2}{|c|}{ AUTOESTIMA } & \multirow[b]{2}{*}{$\begin{array}{l}\text { NIVEL } \\
\text { ALTO }\end{array}$} \\
\hline & $\begin{array}{l}\text { NIVEL } \\
\text { BAJO }\end{array}$ & $\begin{array}{l}\text { NIVEL } \\
\text { MEDIO }\end{array}$ & $\begin{array}{l}\text { NIVEL } \\
\text { ALTO }\end{array}$ & $\begin{array}{l}\text { NIVEL } \\
\text { BAJO }\end{array}$ & $\begin{array}{l}\text { NIVEL } \\
\text { MEDIO }\end{array}$ & $\begin{array}{l}\text { NIVEL } \\
\text { ALTO }\end{array}$ & $\begin{array}{l}\text { NIVEL } \\
\text { BAJO }\end{array}$ & $\begin{array}{l}\text { NIVEL } \\
\text { MEDIO }\end{array}$ & \\
\hline Varón & 19 & 29 & 1 & 10 & 30 & 9 & 1 & 7 & 41 \\
\hline Mujer & 17 & 25 & 6 & 6 & 29 & 13 & 0 & 14 & 34 \\
\hline$\overline{\text { Total }}$ & 36 & 54 & 7 & 16 & 59 & 22 & 1 & 21 & 75 \\
\hline
\end{tabular}

Respecto a la edad los estadísticos de contraste ponen de manifiesto que no existen diferencias significativas entre la edad y los niveles de autoestima, pero por el contrario los niveles de ansiedad en cualquiera de sus modalidades varían significativamente en función de la edad (Tabla 3). De modo que, conforme el alumnado aumenta en edad, aumenta también la probabilidad de incrementar sus niveles de ansiedad estado (tabla 4), y mucho más los niveles de ansiedad rasgo (tabla 5).

\begin{tabular}{llll}
\hline \multicolumn{4}{c}{ Tabla 3. Estadísticos de contrate. Prueba de Kruskal-Wallis con la variable edad. } \\
\hline & STAI_AE_AGRU & STAI_AR_AGRU & ROSEMBER_AGRU \\
\hline Chi-cuadrado & 22,483 & 13,022 & 1,642 \\
\hline gl & 4 & 4 & 4 \\
\hline Sig. asintót. &, 000 &, 011 &, 801 \\
\hline
\end{tabular}

Tabla 4. Distribución de la muestra en función de la ansiedad-estado y la edad. Tabla de contingencia

\begin{tabular}{llllll}
\hline & & \multicolumn{2}{l}{ NIVEL DE ANSIEDAD-ESTADO } & \multirow{2}{*}{ Total } \\
\cline { 3 - 5 } & & NIVEL BAJO & NIVEL MEDIO & NIVEL ALTO & \\
\hline \multirow{4}{*}{ EDAD } & 12 Recuento & 10 & 3 & 0 & 13 \\
& 13 Recuento & 12 & 12 & 0 & 24 \\
& 14 Recuento & 4 & 23 & 1 & 28 \\
& 15 Recuento & 6 & 14 & 6 & 26 \\
& 16 Recuento & 4 & 2 & 0 & 6 \\
\hline Total & Recuento & 36 & 54 & 7 & 97 \\
\hline
\end{tabular}

Tabla 5. Distribución de la muestra en función de la ansiedad-rasgo y la edad. Tabla de contingencia

\begin{tabular}{llllll}
\hline & & \multicolumn{2}{l}{ NIVEL DE ANSIEDAD-RASGO } & & Total \\
\cline { 3 - 6 } & & NIVEL BAJO & NIVEL MEDIO & NIVEL ALTO & \\
\hline \multirow{4}{*}{ EDAD } & 12 Recuento & 3 & 10 & 0 & 13 \\
& 13 Recuento & 8 & 13 & 3 & 24 \\
& 14 Recuento & 3 & 16 & 9 & 28 \\
& 15 Recuento & 2 & 17 & 7 & 26 \\
Total & 16 Recuento & 0 & 3 & 3 & 6 \\
& Recuento & 16 & 59 & 22 & 97
\end{tabular}

El último de los objetivos se plantea conocer la relación entre la ansiedad y la autoestima, para ello se ha recurrido a la estadística inferencial de contraste, y se ha comprobado que existe relación entre la autoestima y la ansiedad-estado $(p=0,017)$ y con la ansiedad rasgo $(p=0,002)$. Concretamente, se observa en los valores promedio 
que cuanto mayor es el nivel de autoestima menor es el nivel de ansiedad estado, incrementándose esa diferencia en la ansiedad rasgo (tabla 6).

\begin{tabular}{|c|c|c|c|c|}
\hline & ROSEMBER_AGRU & $\mathbf{N}$ & Rango promedio & Suma de rangos \\
\hline \multirow{3}{*}{ STAI_AE_AGRU } & NIVEL MEDIO & 21 & 61,67 & 1295,00 \\
\hline & NIVEL ALTO & 75 & 44,81 & 3361,00 \\
\hline & Total & 96 & & \\
\hline \multirow{3}{*}{ STAI_AR_AGRU } & NIVEL MEDIO & 21 & 64,69 & 1358,50 \\
\hline & NIVEL ALTO & 75 & 43,97 & 3297,50 \\
\hline & Total & 96 & & \\
\hline
\end{tabular}

\section{Discusión}

La ansiedad es una temática bastante estudiada entre los escolares, aunque en menor proporción, si la asociamos a otros constructos como la autoestima. El presente estudio ha sido realizado con el objetivo de conocer la relación que existe entre ansiedad y autoestima en los adolescentes. En general, los resultados obtenidos muestran que los participantes obtuvieron niveles altos de autoestima y niveles medios de ansiedad, siendo más elevados en la ansiedad rasgo frente a la ansiedad estado.

A nivel descriptivo, y en consonancia con lo estipulado en el primer objetivo, se observa que a pesar de no existir diferencias significativas en función del género, la autoestima alcanza valores superiores en hombres que en mujeres. Esta diferencia de género en la autoestima ha sido puesta de manifiesto en estudios precedentes de forma divergente. Trabajos, como los de Au (1995) y Rothenberg (1997), señalan que la evolución de la autoestima depende en gran medida de las diferencias individuales, presentando los chicos niveles de autoconcepto ligeramente superiores a las chicas. Además, ellas muestran un autoconcepto global más bajo y un peor autoconcepto físico (Orenstein, 1994), pero tienen puntuaciones superiores en cuanto a competencia social, habilidades sociales y adaptación social (Crain, 1996), de ahí que presenten un autoconcepto social y emocional más elevado que los chicos (Amezcua y Pichardo, 2000). Garaigordobil, Durá y Pérez (2005) y Pastor, Balaguer y García-Merita (2003), obtienen resultados similares donde encuentran que los alumnos presentan una mayor autoestima que las alumnas y mayor autoconcepto. Sin embargo, otros autores encuentran que las niñas tienen una autoestima ligeramente más elevada (Couoh Lope, Góngora Huchim, García Rivero,Rafael y Olmos Barragán, 2015; Flores, Cortes y Góngora, 2008). Mora y Raich (2005), señalan como posible explicación de estas diferencias de género, que el sexo femenino le da mas importancia a la valoración, la aceptación o el rechazo que reciban, mostrando mayor preocupación por ser aceptadas socialmente y por comportarse de acuerdo a las normas sociales.

Por otra parte, no se han obtenido diferencias significativas entre la edad y los diferentes niveles de autoestima, coincidiendo con los resultados de Pastor, Balaguer y García-Merita (2014) quienes encuentran que no existen diferencias en autoestima en función del curso académico, en un grupo de adolescentes valencianos. Sin embargo, Vicent et al., (2015) si que reportan una tendencia de los alumnos de mayor edad a puntuar más alto en autoestima que sus iguales de menor edad. Marsh (1989), a través de un metaanálisis, encontró que las puntuaciones en Apariencia Física, Habilidades Físicas, Relaciones con el Mismo Sexo, Sinceridad/Veracidad, 
Autoestima, Matemáticas y Autoconcepto Académico General, fueron superiores en $7^{\circ}$ grado (12 a 13 años), produciéndose un descenso en $8^{\circ}$ y $9^{\circ}$ grado (13 a 15 años) y un aumento en los cursos de $10^{\circ}$ a $11^{\circ}$ (15 a 17 años). Estudios llevados a cabo en distintos países (Lee, Super, y Harkness, 2003; Muris, Meesters, y Fijen, 2003), muestran que los niños más pequeños tienden a presentar una autopercepción más positiva que los niños mayores. Por tanto, los resultados existentes son dispares.

Si nos centramos en el nivel de ansiedad estado, vemos como, de manera general, el alumnado estudiado muestra un nivel medio, seguido de un nivel bajo. Sin embargo, aunque sigue predominando el nivel medio en el nivel de ansiedad rasgo, el porcentaje de alumnos que obtienen una ansiedad elevada es alto $(22,68 \%)$. Por tanto, podemos concluir que, a rasgos generales, el nivel de ansiedad en nuestros alumnos y alumnas es medio, y que ambos niveles de ansiedad son mayores en mujeres que en hombres, en nuestra muestra. Estos resultados concuerdan con los de Contreras, et al (2005), que reflejaron que el nivel de ansiedad en esta etapa correspondía a un nivel medio y con los de Lockrer y Cropley (2004) y García-Fernández, Inglés, MartínezMonteagudo, Marzo y Estévez (2011), quienes valoraron que dicho nivel también era mayor en mujeres que en hombres. Encontramos además, que el nivel de ansiedadrasgo es mayor que el de ansiedad-estado.

Finalmente, teniendo en cuenta las variables del estudio, observamos la existencia de una relación negativa entre la autoestima y la ansiedad estado y ansiedad rasgo. En concreto, a mayor autoestima, menores niveles en las otras variables, siendo algo menor la ansiedad rasgo. Esto coincide con lo encontrado por Peñaloza (2015), que observa correlación negativa entre dichos constructos, en una muestra de alumnos de primaria y secundaria. Observamos también como en el caso de los varones presentan un nivel de ansiedad más bajo y una autoestima mayor, mientras que con las alumnas ocurre lo contrario, a mayor nivel de ansiedad, menor nivel de autoestima, por lo que en ambos niveles se muestra una correlación inversa, en mayor o menor medida.

En conclusión, por un lado, observamos que el nivel de autoestima de la muestra es alto, siendo dicho porcentaje mayor en alumnos que en alumnas. Por otro lado, el nivel de ansiedad estado y ansiedad rasgo analizado corresponde a un nivel medio, aunque observamos una distinción en función del género, siendo este nivel mayor en las alumnas. Por tanto, los varones presentan un nivel de ansiedad mas bajo y una autoestima mayor y ellas, mayores niveles de ansiedad y menores de autoestima. Por último, encontramos una relación significativa y negativa entre las dos variables de estudio, ansiedad y autoestima, ya que, a mayor nivel de autoestima menor nivel de ansiedad.

\section{Referencias}

Aldana, K. (2012). Programa de apoyo a la autoestima para niños y niñas victimas de Bullying. Tesis Doctoral. Guatemala: Universidad Rafael Landivar.

Au, R. C. P. (1995). Academic failure and learned hopelessness in Hong Kong academically low achievers. Bulletin of the Hong Kong Psychological Society, 34 (35), 83-100.

Amezcua, J. A. \& Pichardo, M.C. (2000). Diferencias de género en autoconcepto en sujetos adolescentes. Anales de Psicología, $16,2,207-214$.
Bobes, J. (2001). Trastornos de ansiedad y trastornos depresivos en atención primaria. Barcelona: Masson.

Bolivar Botía, A. (2006). Familia y escuela: dos mundos llamados a trabajar en común. Revista de educación, (339), 119-146.

Branden, N. (1993). El poder de la autoestima. Como potenciar este importante recurso psicológico. México: Paidos. 
Branden, N. (1995). Los seis pilares de la autoestima. El libro definitivo sobre la autoestima por el más importante especialista en la materia. México: Paidos.

Cándido Corral, A., Díaz Castro, C., Duque Aparicio, C., Estévez Ortega, E., \& Gamero Larios, L. J. (2016). Influencia de la jubilación en la satisfacción con la vida y la autoestima. Reidocrea. Monografico Psicología y Salud. 21-26.

Ceballos Garinay, H. (2003) El saber artístico. México: Coyoacan.

Ciarrochi, J., Chan, A., \& Bajgar, J. (2001). Measuring emotional intelligence in adolescents. Personality and Individual Diferrences. 31, 1105-1119.

Contreras, et. al (2005). Autoeficacia, ansiedad y rendimiento académico en adolescentes. Facultad de psicología. Universidad de Santo Tomás. Recuperado de: http://www.scielo.org.co/pdf/dpp/v1n2/v1n2a07.pdf

Couoh Lope, C. L., Góngora Huchim, A., García Rivero, A. D., Rafael, I., y Olmos Barragán, N. A. (2015). Ansiedad y autoestima en escolares de educación primaria de Mérida, Yucatán. Enseñanza e Investigación en Psicología, 20(3), 1-

Crain, M. (1996). The influence of age, race and gender on child and adolescent self-concept. En B. A. Bracken (Ed.), Handbook of self-concept,. New York: Wiley, 395-420.

Domingo Roget, Á., \& Verdera Albiñana, A. (2010). La formación competencial del niño y la niña: bases para una propuesta articulada entre familia y escuela. Infancias Imágenes, 9(2), 6-23.

Endler, N.S. (1975). A person-situation interaction model for anxiety. A person-situation interaction model for anxiety. En Cano, A., y Miguel, J. J. (1999). Evaluación de la ansiedad desde un enfoque interactivo y multidimensional: El inventario de Situaciones y Respuestas de Ansiedad (ISRA). Psicología Contemporánea, 6, 1, 14-21.

Erikson. (1968). Identidad, juventud y crisis. Buenos Aires: Paidos.

Fernández-Berrocal, P., \& Extremera, N. (2002). La inteligencia emocional como una habilidad esencial en la escuela. Revista Iberoamericana de Educación, 29, 1-6

Flores, M., Cortés, L., \& Góngora, E. (2008). Familia, crianza y personalidad: una perspectiva etnopsicológica. Mérida (México): UADY.

Garaigordobil, M., Durá, A., \& Pérez, J.I. (2005). Síntomas patológicos, problemas de conducta y autoconceptoautoestima: un estudio con adolescentes de 14 a 17 años. Anuario de psicología clínica y salud, 1, 53-63.

García-Fernández, J. M., Inglés, C. J., Martínez-Monteagudo, M. C., Marzo, J. C., \& Estévez, E. (2011). Inventario de Ansiedad Escolar: Validación en una muestra de estudiantes de educación secundaria. Psicothema, 23 (2), 301-307.

González-Pienda, J. A., Nuñez Pérez, J. C., Glez Pumariega, S., \& García García, M. S. (1997). Autoconcepto, autoestima y aprendizaje escolar. Psicothema, 9(2), 271-289.
González, M.C., \& Tourón, J. (1992). Autoconcepto y rendimiento académico. Sus implicaciones en la motivación y en la autorregulación del aprendizaje. Pamplona: EUNSA.

Jadue, G. (2001). Algunos efectos de la ansiedad en el rendimiento escolar. Estudios pedagógicos (Valdivia), (27), $111-118$.

Kashani, J., \& Orvaschel, H. (1990). A Community Study of Anxiety in Children and Adolescents. American Journal of Psychiatry, 147, 313-318.

Kernis, M.H. (2003). Toward a conceptualization of optimal selfesteem. Psychological Inquiry, 14, 1-26.

Lee, J., Super, C. M., \& Harkness, S. (2003). Self-perception of competence in Korean children: Age, sex and home influences. Journal of Social Psychology, 6, 133-147.

Locker, J., \& Cropley, M. (2004). Anxiety, depression and selfesteem in Secondary school children. School Psychology International, 25, 3, 333-345.

Marsh, H. W. (1989). Age and sex effects in multiple dimensions of self-concept: Preadolescence to early adulthood. Journal of Educational Psychology, 81, 417-430.

Montoya Flores, B. I., \& Landero Hernández, R. (2013). Satisfacción con la vida y autoestima en jóvenes de familias monoparentales y biparentales. Psicología y Salud, 18(1), 117-122.

Mora, M., \& Raich, R. (2005). Autoestima: Evaluación y tratamiento. Madrid: Síntesis.

Muris, P., Meesters, C., \& Fijen, P. (2003). The Self-Perception Profile for Children: further evidence for its factor structure, reliability, and validity. Personality and Individual Differences, $35,1791-1802$

Musitu, G. (1996). Proyecto autoestima. Educación familiar y socializaicón de los hijos. Barcelona: Idea Books.

Orenstein, Peggy. (1994). Schoolgirls: young women, self-esteem, and the confidence gap. Nueva York: Doubleday.

Pastor, Y., Balaguer, I., \& García-Merita, M. L. (2003). El autoconcepto y la autoestima en la adolescencia media: análisis diferencial por curso y género. Revista de psicología social,18 (2), 141-159.

Peñaloza Luna, L. K. (2015). Ansiedad y autoestima en la niñez intemedia en alumnos de primaria y secundaria. Tesis de licenciatura. Lima: Universidad femenina del sagrado corazón.

Rodríguez, C., \& Caño, A. (2012). Autoestima en la adolescencia: análisis y estrategias de intervención. Internacional Journal of Psychology an Psychological Therapy, 12, 3, 389-403.

Rosenberg, M. (1965). La autoimagen del adolescente y la sociedad. Buenos Aires: Paidós (traducción de 1973).

Rothenberg, D. (1997). Supporting Girls in Early Adolescence. Washington, DC: Office of Educational Research and Imporvement. 
Sierra, J.C., Ortega, V., \& Zubeidat, I. (2003). Ansiedad, angustia y estrés: tres conceptos a diferenciar. Revista mal-estar e subjetividade- Fortaleza, 1, 3, 10-59.
Smelser, N.J. (1989). Self-esteem and social problems: an introduction. En Mecca, A. M. et alii (eds.: The social importance of self-estem (294-326). University of California Press, Berkeley.

Vicent, M., Lagos-San Martín, N., Gonzálvez, C., Inglés, C. J., García-Fernádez, J.M., \& Gomis, N. (2015). Diferencias de género y edad en autoconcepto en estudiantes chilenos. Revista de psicología, 24(1), 1-16. 